\title{
Measurement of the neutron capture resonances for platinum using the Ge spectrometer and pulsed neutron beam at the J-PARC/MLFIANNRI
}

\author{
Koichi Kino $^{1, \text { a }}$, Hiroyuki Hasemi ${ }^{2}$, Atsushi Kimura ${ }^{3}$, and Yoshiaki Kiyanagi ${ }^{4}$ \\ 1 National Institute of Advanced Industrial Science and Technology, Research Institute for Measurement and Analytical \\ Instrumentation, 1-1-1 Umezono Tsukuba Ibaraki, Japan \\ ${ }^{2}$ Hokkaido University, Faculty of Engineering, Kita13 Nishi8 Kita-ku Sapporo, Japan \\ 3 Japan Atomic Energy Agency, 2-4 Shirakata Shirane Tokai Naka Ibaraki, Japan \\ ${ }^{4}$ Nagoya University, Graduate School of Engineering, Furo-cho Chikusa-ku Nagoya, Japan
}

\begin{abstract}
The neutron capture cross-section for platinum was measured at J-PARC/MLF/ANNRI. The intense pulsed neutron beam was impinging on a natural platinum foil sample and the emitted prompt $\gamma$-rays were detected by a Ge spectrometer. The peak energies of the low energy resonances for natural platinum are consistent with those of the JEFF-3.1.2, RUSFOND2010 and next-JENDL data libraries except for the $20-\mathrm{eV}$ resonance. The resonance cross-sections of the next-JENDL library do not contradict the present measurements within the uncertainty of the absolute value of the present work. We analysed the prompt $\gamma$-ray spectrum and found a clear $7921.93 \mathrm{keV}$ peak that originates from the transition from the ${ }^{196} \mathrm{Pt}$ compound state to its ground state. The neutron capture cross-section for ${ }^{195} \mathrm{Pt}$ was obtained by choosing events of this peak. The peak energies of most of the low energy resonances are almost consistent with those of the RUSFOND2010 and next-JENDL libraries. However, there was a disagreement for the $20-\mathrm{eV}$ resonance.
\end{abstract}

\section{Introduction}

Platinum is one of the most important elements in our society and is widely used as a catalyst. For example, it is used to purify car exhaust gases and generate electricity in fuel cells. However, in car exhaust applications platinum's activity as a catalyst deteriorates due to coupling to sulphur compounds. In the case of the fuel cell, deterioration occurs due to coupling to carbon monoxide. In order to investigate and improve these applications, non-destructive imaging methods may be useful. One such method is neutron resonance absorption spectroscopy (N-RAS) $[1,2]$. This method is based on the irradiation of a sample by a pulsed neutron beam. By using a position sensitive neutron detector, atomic density and temperature can be obtained as images.

The N-RAS needs resonance parameters of the nuclei included in the experimental sample. However, experimental data of resonance spectra for platinum is poor in the low energy resonance region. There are two data sets in the EXFOR library [3] of total cross sections for natural platinum. Both data sets show resonances at almost the same neutron energies. However, there are discrepancies in the absolute values of the resonances. For the capture cross section, no spectral data is available below $200 \mathrm{eV}$. For the nuclear data library, no library provides resonance parameters, although Mughabghab compiles the resonance parameters by referring the values in old papers [4]. JEFF-3.1.2 [5], JEFF-3.2 [6] and RUSFOND2010 [7] provide resonance spectra as a function of energy. On the

a e-mail: koichi.kino@aist.or.jp other hand, JENDL aims at a complete library by adding elements whose importance is not high for nuclear energy applications. Very recently, K. Shibata published an article of the evaluation on platinum for the next version of the JENDL general-purpose file [8].

The purpose of this work is to measure the low energy resonances for platinum experimentally. As a first step, we performed a capture cross-section measurement using the Accurate Neutron-Nucleus Reaction Measurement Instrument (ANNRI) [9] in the Material and Lifescience Experimental Facility (MLF) at the Japan Proton Accelerator Research Complex (J-PARC).

\section{Experiment and data analysis}

We used the J-PARC/MLF/ANNRI [9]. An intense pulsed neutron beam was impinging on a natural platinum foil sample at a distance of $21.5 \mathrm{~m}$ from the neutron source. The size of the sample is $50 \mathrm{~mm}$ square with a thickness of $2.5 \mu \mathrm{m}$ and a purity of $99.9 \%$. The neutron beam is collimated by a rotary collimator [10] and has a diameter of $22 \mathrm{~mm}$ at the sample position. Prompt $\gamma$-rays following neutron capture at the sample were observed by $\mathrm{Ge}$ spectrometers set above and below the sample. The spectrometers consisted of $14 \mathrm{Ge}$ crystals in total. The neutron energy of each event was determined by the time of flight (TOF) method between the neutron source and sample. In order to reduce $\gamma$-rays from the neutron source, a lead filter with a thickness of $37.5 \mathrm{~mm}$ was set on the neutron beam line. The proton beam power of the

(C) The Authors, published by EDP Sciences. This is an Open Access article distributed under the terms of the Creative Commons Attribution License 4.0 (http://creativecommons.org/licenses/by/4.0/). 


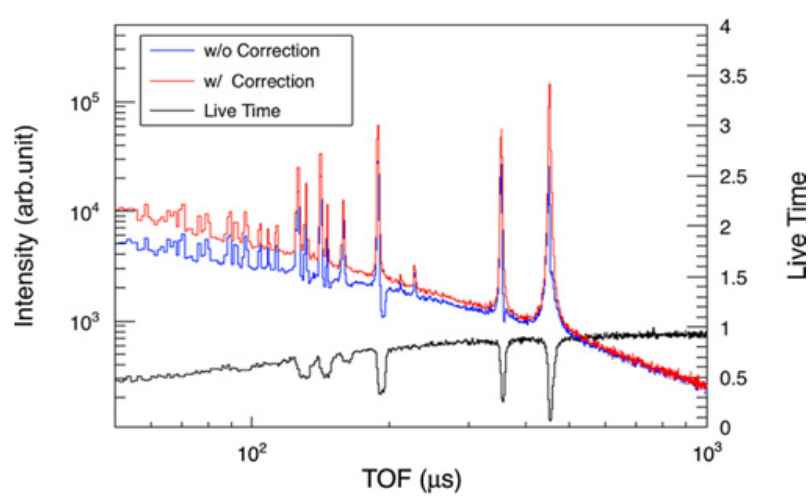

Figure 1. TOF spectrum without and with the dead time correction and live time spectrum. The dips near the resonance peaks, which are due to the dead time of the Ge spectrometer, are corrected.

neutron source was $305 \mathrm{~kW}$ during the experiment. The measurement time of the platinum sample was 31 hours.

The data analysis was conducted mainly by the following three steps. The first is the dead time correction. In order to obtain the live time spectrum as a function of TOF, we put pulse signals randomly with time at a rate of $1.4 \mathrm{kHz}$ into the analogue signal lines of the Ge spectrometer. The data originating from these signals were distinguished by their pulse height [11]. The raw spectrum was divided by the live time spectrum. Figure 1 shows the TOF spectrum before and after the correction and the live time spectrum. This correction is important especially around the resonance peaks due to the high counting rate due to the large cross section of the capture reaction. The second step is a background subtraction. The background spectrum was obtained by the open beam data. Finally, the last step is the division by the neutron flux spectrum, which is a function of TOF. The flux spectrum was obtained using a boron sample. The size of the sample is $10 \mathrm{~mm}$ in diameter with a weight of $96.12 \mathrm{mg}$. The isotopic ratio of ${ }^{10} \mathrm{~B}$ is $90.4 \%$. The neutron flux spectrum was calculated from the experimental data using the $478 \mathrm{keV} \gamma$-ray following the ${ }^{10} \mathrm{~B}(\mathrm{n}, \alpha)^{7} \mathrm{Li}$ reaction and the cross section of this reaction. The dependency of neutron beam on TOF was taken into account by the division with the neutron flux spectrum.

\section{Discussions}

Figures 2 and 3 show the capture cross-section spectra for natural platinum in wide and narrow energy regions. The cross section was normalized to the value (10.3 barn) reported by Mughabghab [4] at $25.3 \mathrm{meV}$. The deviation from the 1/v law seen in Fig. 2 might be due to some kind of constant background and it is about 1 barn. This gives an uncertainty of about $10 \%$ for the absolute value of the cross section.

Figure 2 shows that the energies of major low energy resonances are almost in agreement with those of JEFF3.1.2 and RUSFOND2010. The blue line is the spectrum converted at $300 \mathrm{~K}$ from the next-JENDL evaluation resonance parameters [8]. The resonance parameters of this evaluation are taken from the compilation work of Mughabghab. The experimental data showed agreement in resonance energies with the next-JENDL evaluation except for the resonance at $20 \mathrm{eV}$. The disagreement of the $20-\mathrm{eV}$

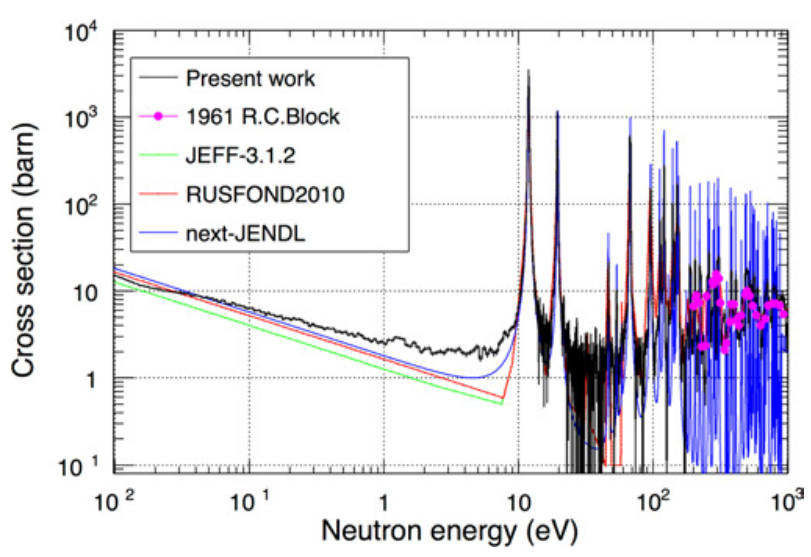

Figure 2. Neutron capture cross-section spectrum for natural platinum in a wide energy region. The cross section was normalized to the value (10.3 barn) reported by Mughabghab [4] at $25.3 \mathrm{meV}$.

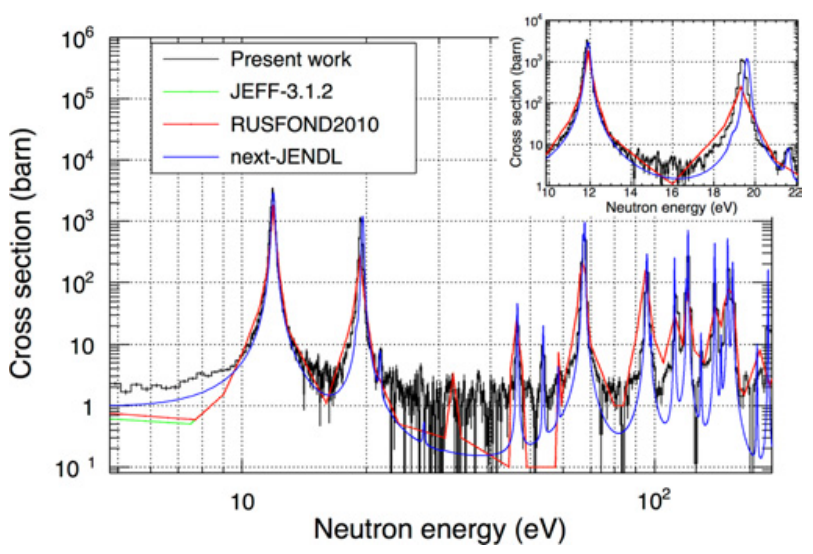

Figure 3. Neutron capture cross-section spectrum for natural platinum in a narrow energy region. The spectra of JEFF-3.1.2 and RUSFOND2010 almost overlap in this energy region.

resonance energy is clearly seen in Fig. 3. The spectrum above $200 \mathrm{eV}$ is almost in agreement with the experimental data by R.C. Bloch. The cross sections at the low energy resonance peaks given by JEFF-3.1.2 and RUSFOND2010 were smaller than our experimental data in Fig. 3 even though the experimental data has a $10 \%$ uncertainty. On the other hand, the cross sections of the resonances by the next-JENDL did not contradict those of the present data.

The ANNRI has an advantage that the $\gamma$-ray energy is precisely determined by the Ge spectrometer. Therefore, we made use of it for selecting platinum isotopes in the capture reaction. There are six stable isotopes of platinum. The neutron separation energies are different for each isotope. Therefore, one can distinguish isotopes by selecting primary $\gamma$-ray on the basis of the decay from the compound state after neutron capture reaction to the ground state. Figure 4 shows the $\gamma$-ray energy spectrum in the energy region from 5500 to $8000 \mathrm{keV}$. There are many photoelectron peaks and the arrows in the figure indicate single-neutron separation energies for platinum isotopes. The peak corresponding to ${ }^{196} \mathrm{Pt}$ is seen very clearly at $7921.93 \mathrm{keV}$. Some peaks for other isotopes are also seen although the signal to noise ratio is not high. Therefore, we applied the isotope separation only on ${ }^{196} \mathrm{Pt}$. This means that we select neutron capture reaction for ${ }^{195} \mathrm{Pt}$. The natural abundance of ${ }^{195} \mathrm{Pt}$ is $33.78 \%$. 


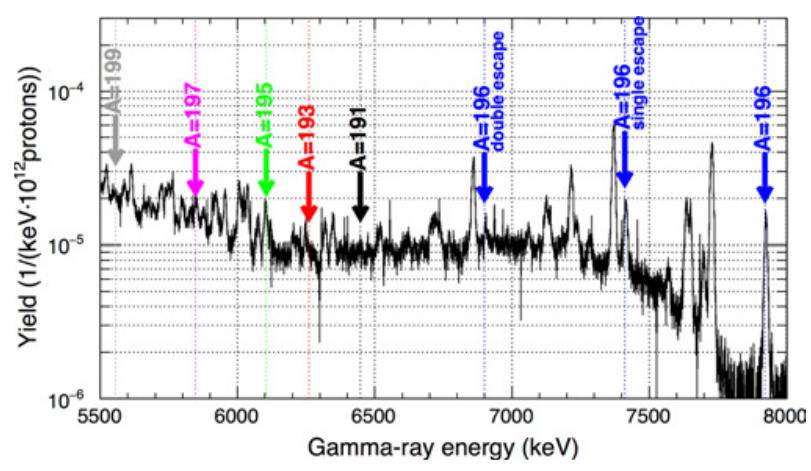

Figure 4. Energy spectrum of prompt $\gamma$-rays in the energy region from 5500 to $8000 \mathrm{keV}$. The arrows indicate single neutron separation energies for platinum isotopes.

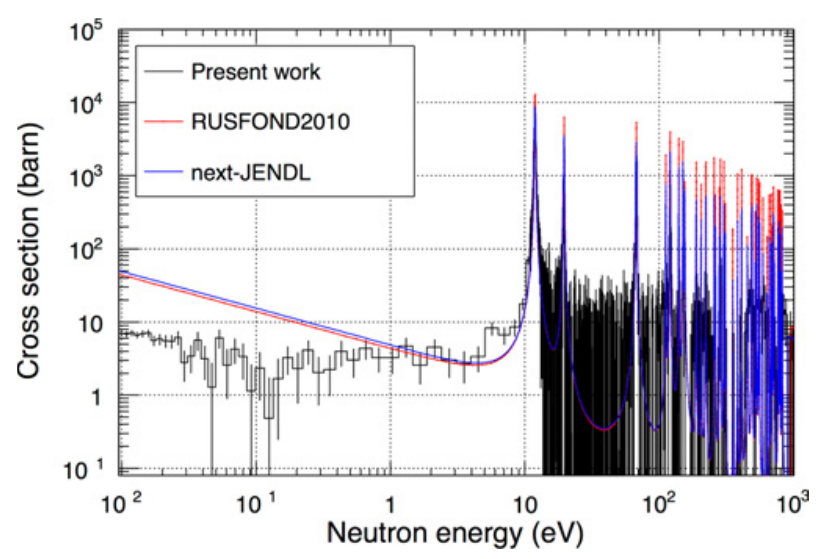

Figure 5. Neutron capture cross-section spectrum for ${ }^{195} \mathrm{Pt}$ in a wide energy region.

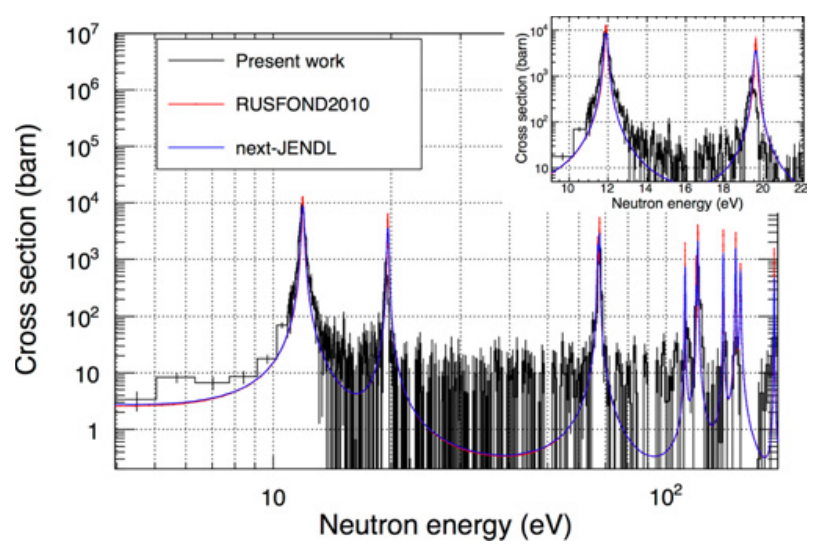

Figure 6. Neutron capture cross-section spectrum for ${ }^{195} \mathrm{Pt}$ in a narrow energy region.

Figures 5 and 6 show the capture cross-section spectra for ${ }^{195} \mathrm{Pt}$ in wide and narrow energy regions. The normalization was performed by setting the cross section of the present work at $12-\mathrm{eV}$ resonance to the value given by the next-JENDL library. The resonances, whose energies are 46 and $95 \mathrm{eV}$ for example, disappeared after choosing ${ }^{195} \mathrm{Pt}$. This is consistent with RUSFOND2010 and next-JENDL. Disagreement of resonance energy for the $20-\mathrm{eV}$ resonance is obtained between the present work and next-JENDL as shown in Fig. 6. In general, the decay schemes are different depending on the neutron capture states. This could be the reason for the disagreements of the cross sections between the present work and nuclear data libraries at the thermal neutron energy as seen in Fig. 5 and the 20-eV resonance as seen in Fig. 6.

\section{Summary}

The neutron capture cross-section for platinum was measured at J-PARC/MLF/ANNRI. For natural platinum, most of the measured resonances were found at almost the same energies as the various nuclear data libraries except for the $20-\mathrm{eV}$ resonance. The cross sections of the low energy resonances of the present work did not contradict those given by the next-JENDL library. We obtained the capture cross-section spectrum for ${ }^{195} \mathrm{Pt}$ by choosing the prompt $\gamma$-rays for this isotope. Most of the resonance energies of the present work were consistent with those of RUSFOND2010 and next-JENDL libraries. However, the energy of the $20-\mathrm{eV}$ resonance was different between the present work and the nuclear data libraries.

This work was performed using the experimental instruments at the MLF at J-PARC and supported by the J-PARC staff. The authors would like to thank Dr. K. Shibata and Dr. N. Iwamoto for providing the nuclear data for the next version of JENDL.

\section{References}

[1] H. Sato, T. Kamiyama, Y. Kiyanagi, Nucl. Instr. and Meth. A 605, 36 (2009)

[2] H. Hasemi, M. Harada, T. Kai, et al., Nucl. Instr. and Meth. A 773, 137 (2015)

[3] N. Otsuka, E. Dupont, V. Semkova, et al., Nucl. Data Sheets 120, 272 (2014)

[4] S.F. Mughabghab, Atlas of neutron resonances (Elsevier, Amsterdam, 2006)

[5] JEFF-3.1.2, http://www .oecd-nea.org/dbforms/ data/ eva/evatapes/jeff_31/JEFF312/

[6] JEFF-3.2, http://www.oecd-nea.org/dbforms/ data/eva/evatapes/jeff_32/

[7] RUSFOND2010, http://www.ippe.ru/podr/ abbn/english/libr/rosfond.php

[8] K. Shibata, J. Nucl. Sci. Technol 54, 147, (2016)

[9] M. Igashira, Y. Kiyanagi, M. Oshima, Nucl. Instr. and Meth. A 600, 332 (2009)

[10] K. Kino, M. Furusaka, F. Hiraga, Nucl. Instr. and Meth. A 626-627, 58 (2011)

[11] T. Kin, K. Furutaka, S. Goko, et al., J. Korean Phys. Soc. 59, 1769 (2011) 